from stepwise heating experiments on bulk samples. This type of experiment provides a quantum jump over simple bulk analysis in the detailed knowledge one obtains from a sample. The state of the art now, however, requires a further quantum jump in information content per experiment, namely stepwise heating experiments on various discrete mineral phases or assemblages from a meteorite. It would seem particularly important to do rare gas analyses of at least neon and xenon in a given sample, and to have oxygen and magnesium isotope analyses done on aliquots of the rare gas samples. The questions are: do $\mathrm{R}$ xenon, neon $\mathrm{E}$ (ref. 15) and the oxygen and magnesium isotope anomalies occur together, with what frequency and in what minerals? Are $\mathbf{P}$ xenon and $\mathrm{R}$ xenon well mixed, and if so is it on a microscopic scale (individual mineral phases and/or grains) or a macroscopic (in bulk) scale?

I thank Drs A. G. W. Cameron, R. O. Pepin and D. L. Phinney for stimulating conversations.

University of London Observatory,

Mill Hill Park,

London NW7 2QS, UK

*Permanent address: Theoretical Studies Branch, Space Science Division, NASAAmes Research Center, Moffett Field, California 94035.

Received September 27; revised December 13, 1974.

1 Phinney, D. L. Meteoritics, 4 (1973).

Manuel, O. K., Henneke, E. W., and Sabu, D. D., Nature, 240, 99 (1972).

Alexander, E. C. Jr, Lewis, R. S., Reynolds, J. H., and Michel, M. C., Science, 172, 837 (1971).

Reynolds, J. H., and Turner, G., J. geophys. Res., 69, 3263 (1964).

Hoffman, D. C., and Hoffman, M. M., A. Rev. nucl. Sci., 24 (in the press).

Anders, E., and Heymann, D, Science, 164, 821 (1969).

Rao, M. N., Nucl. Phys., A, 140, 69 (1970), 1972).

Takaota, N., Mass Spectroscopy, 20, 287 (1972).

10 Pepin, R. O., in Origin and Distribution of the Elements, 379 (Pergamon, Oxford, 1968

Eugster, O., Eberhardt, P., and Geiss, J., Earth planet Sci. Lett., 1, 99 (1966).

12 Marti, K., Meteoritics, 5, 208 (1970).

13 Sabu, D. D. Henneke, E. W. and Manuel, O. K., Nature, 251, 21 (1974).

4 Black, D. C, and Pepin, R. O., Earth planet. Sci. Lett., 6, 395 (1969).

15 Black, D. C., Geochim. cosmochim. Acta, 36, 377 (1972).

16 Clayton, R. N., Grossman, L., and Mayeda, T. K., Science, 182, 485 (1973).

17 Gray, C. M., and Compston, W., Nature, 251, 495 (1974)

18 Lee, T., and Papanastassiou, D. A., Geophys. Res. Lett., 1, 227 (1974).

19 Cameron, A. G. W., and Pine, M. R., Icarus, 18, 377 (1973).

20 Cameron, A. G. W., Space Sci. Rev., 15, 121 (1973).

12 Hoyle, F., and Fowler, W. A., Nature, 241, 384 (1973)

22 Black, D. C., in On the Origin of the Solar System, 236 (CNRS, Paris, 1972).

\section{The beginning of a new cycle of solar activity}

ON November 15 , a high latitude sunspot marked the beginning of a new cycle of solar activity. The present cycle is on the decline and is approaching its minimum, a new cycle thus starting before the old one has completely disappeared. At this time the sunspots belonging to the old cycle originate near the equator, whereas those of the new one occur at higher latitudes.

The latitude of the sunspot observed was $+37^{\circ}$, and it was $60^{\circ}$ from the central meridian. It could be seen clearly the following day but not on November 17, when it crossed the western limb. The first spots of a new cycle usually appear more than a year before the coming minimum, so because the present activity is still relatively high, the minimum cannot be expected to occur before 1976 .

Swiss Federal Observatory,

M. WALDMEIER

Schmelzbergstrasse 25 ,

CH 8006 Zürich, Switzerland

Received November 27, 1974.

\section{Mathematical prediction of climatic change}

A WIDESPREAD and increasing degree of attention is being paid to the problem of understanding the physical factors which lead to climatic change. Analysis of past weather data ${ }^{1}$ derived from a wide variety of sources has shown how the main characteristics of climate have changed over recent centuries. If, however, scientific theories are to be developed, it is desirable that mathematical models of climate be constructed. These must include at least the primary global scale dynamics of the atmosphere and should allow the operation of necessary long time-period integrations on available computers.

The primary physical factor responsible for initiating the stirring of the atmosphere into its ever changing assembly of the cyclonic waves and anticyclonic systems is the meridional distribution of solar radiation, $S(\theta)$, where $\theta$ is the latitude. The response of the atmosphere to a given $S(\theta)$ and to the associated requirement of balancing the heat budget is seen in the continuously created train of weather systems which strive to rectify the imbalance; these systems are important agencies in determining climate. The sensitivity of climate to changes in solar radiation or changes in atmospheric transparencies should, therefore, be investigated.

In approximate calculations Budyko ${ }^{2}$ used a system of empirical equations describing an assumed static balance (in a temporal sense) between the residual vertically integrated and zonally averaged annual radiation heating at a given latitude and the vertically integrated meridional flux divergence of heat. He assumed that his empirical heating functions were still valid when the total solar radiation was altered by a series of small percentages. An iterative computer procedure allowed Budyko to calculate the associated shift in the meridional distribution of mean atmospheric temperature, atmospheric and surface albedo, and the new mean, zonally averaged, simulated ice edge position. The primary atmospheric physics on a global scale is contained in Budyko's model (although rather crudely) but he assumed a static atmospheric balance, purporting to represent an asymptotic, annual average situation, corresponding to a prescribed solar heating; and there is no indication of how sensitively the calculated results depend on the mathematical forms of the empirical functions used. Consequently, his main result, suggesting that a $1.5 \%$ reduction in incoming solar radiation (or an equivalent change in atmospheric transparency) would lead to a new ice age, has met with scepticism. An extended form ${ }^{3}$ of the calculation has indicated that as much as a $5 \%$ reduction in solar radiation would be needed before large scale movement of the ice edge would occur.

These simplified statical calculations, however, tell us nothing about time variations around the mean values of the large scale structure of atmospheric dynamics; indeed it may be completely incorrect to assume a stable balance of the averaged flow system for a perturbation of solar heating greater than some

Fig. 1 Position (latitude) of the zonally averaged simulated ice edge as a function of time over a $200-\mathrm{d}$ period. $a$, Solar heating values representing the present decade; $b$, a $3 \%$ reduction in solar heating.

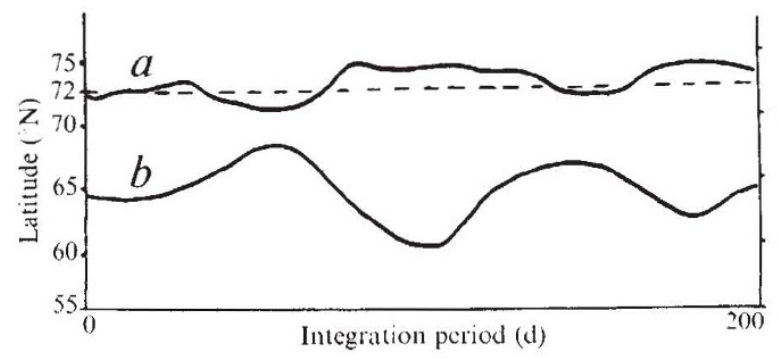

